\title{
Update on Postnatal Corticosteroids to Prevent or Treat Bronchopulmonary Dysplasia
}

\author{
Marco Filippone, MD ${ }^{1}$ Daniel Nardo, MD ${ }^{1} \quad$ Luca Bonadies, MD ${ }^{1}$ Sabrina Salvadori, MD ${ }^{1}$ \\ Eugenio Baraldi, MD ${ }^{1}$ \\ ${ }^{1}$ Neonatal Intensive Care Unit, Department of Woman's and Child's \\ Health, University of Padua, Padova, Italy \\ Am J Perinatol 2019;36(suppl S2):S58-S62.

\begin{abstract}
Address for correspondence Eugenio Baraldi, MD, Neonatal Intensive Care Unit, Department of Woman's and Child's Health, University of Padua, Via Giustiniani 3, Padova 35123, Italy

(e-mail: eugenio.baraldi@unipd.it).
\end{abstract}

\begin{abstract}
Bronchopulmonary dysplasia (BPD) is a major complication of premature birth that significantly affects mortality and long-term morbidity in numerous immature infants. Corticosteroids are particularly suitable for treating BPD, as lung inflammation is central to its pathogenesis. Corticosteroids have considerable, fast beneficial effects on lung function in premature infants with lung disease, but they are also associated with several serious adverse effects, which may have a detrimental impact on long-term outcome. Dexamethasone is the most often used corticosteroid for systemic administration. Despite its value in preventing and treating BPD, its use is associated with several alarming short-term effects and, worst of all, with an increased rate of cerebral palsy in the long term. Dexamethasone nonetheless remains an important therapeutic option for infants with severe lung disease beyond the second to third week of life. Hydrocortisone is an important alternative to dexamethasone, as its use does not appear to be associated with any neurotoxic effects. Its efficacy in the prevention and treatment of BPD has yet to be clearly demonstrated, however. Inhaled corticosteroids might reduce lung inflammation with fewer systemic

\section{Keywords}

- preterm infant

- bronchopulmonary dysplasia

- postnatal corticosteroids adverse effects; however, a recent, large randomized trial showed that inhaled budesonide was associated with an excess mortality, despite its beneficial respiratory effects. In another study, instilling budesonide together with surfactant in the trachea of intubated infants with severe respiratory distress appeared safe and achieved a significant reduction in the rate of BPD at 36 postmenstrual weeks. This important finding needs to be confirmed in a larger trial currently underway.
\end{abstract}

Bronchopulmonary dysplasia (BPD) accounts for considerable mortality, and respiratory and neurodevelopmental morbidity in the most premature infants. ${ }^{1}$ Unfortunately, effective preventive and therapeutic options for BPD are still elusive. $^{2}$

As inflammation is crucial to the pathogenesis of BPD, ${ }^{3}$ corticosteroids (CS)-with their powerful anti-inflammatory properties-are a logical and appealing treatment option for BPD. Over almost four decades of their clinical use, however, CS have revealed not only a clear clinical efficacy but also some alarming adverse effects. A growing awareness of their potentially serious neurotoxicity has drastically curtailed the use of systemic CS (S-CS) treatment for BPD in recent times, after a period of their liberal and widespread use. ${ }^{4}$ They are still commonly used, however, due to their unique effectiveness in facilitating extubation in infants with severe lung disease. ${ }^{5}$

Striking a balance between benefit and harm is not easy in most cases, so the use of CS for BPD remains controversial. Research efforts have focused on identifying the safest preparation (dexamethasone vs. hydrocortisone) and the optimal dosage and timing of systemic treatments. The use of topical administration (inhalation or tracheal instillation) has also attracted considerable interest.

\section{Systemic Corticosteroids}

Most of what is known about the systemic administration of CS to prevent or treat BPD has been condensed in two
Copyright (c) 2019 by Thieme Medical Publishers, Inc., 333 Seventh Avenue, New York, NY 10001, USA. Tel: +1(212) 584-4662. ISSN 0735-1631. 
recently updated Cochrane reviews on early (0-7 days of life $)^{6}$ and later ( $>7$ days of life) S-CS treatment. ${ }^{7}$ Most of the data concern the use of dexamethasone (the preparation traditionally adopted in clinical practice), but the data on hydrocortisone are of special interest because of the conviction that it is potentially safer, based on plausible physiological considerations and preliminary observations. ${ }^{4}$

\section{Early Systemic CS Treatment (0-7 Days of Life)}

The meta-analysis on early S-CS ${ }^{6}$ included 32 randomized controlled trials (RCTs) enrolling 4,395 participants.

Though it did not affect mortality, early dexamethasone had several clear beneficial effects, including lower rates of BPD ( - Table 1). Several serious short-term adverse effects were reported, however, most notably gastrointestinal bleeding and perforation. More importantly, dexamethasone was associated with higher rates of cerebral palsy (CP) and abnormal neurological findings in the longer term (-Table 1). The routine early use of dexamethasone is therefore not recommended, as the benefits do not outweigh the risks.

Data on early hydrocortisone administration were more limited, with low power to reveal beneficial or harmful effects for most comparisons. ${ }^{6}$ Overall, the benefits of hydrocortisone were mainly limited to a slight reduction in the combined outcome death/BPD at 36 weeks postmenstrual age (PMA) (-Table $\mathbf{1}$ ). The main adverse effect was a significantly increased rate of gastrointestinal perforation, while-notably-no neurological problems emerged in the longer term. The Cochrane review concluded that, taking the available data as a whole, the routine use of early hydrocortisone is not recommended, as the risk of gastrointestinal perforation outweighs the marginal benefit for respiratory health.

Interestingly, the meta-analysis included the positive findings of the largest RCT on early hydrocortisone, the PREMILOC trial, ${ }^{8}$ in which low-dose hydrocortisone was administered over the first 10 postnatal days in 523 infants born at 24 to 27 weeks gestational age (GA). Treatment with hydrocortisone resulted in a better BPD-free survival $(p=0.04$, number needed to treat [NNT]: 12$)$, and there were no significant adverse effects, apart from a higher rate of sepsis in the subgroup of infants born at 24 to 25 weeks.

The rates of neurodevelopmental impairment (of any severity) and CP were similar in the hydrocortisone-treated and control groups at 22 months corrected age, ${ }^{9}$ suggesting that low-dose early hydrocortisone has no neurotoxic effects. A further analysis ${ }^{10}$ showed that hydrocortisone was even associated with better overall neurological findings in the subgroup of infants born at 24 to 25 weeks PMA. This result might be influenced by the small size of the group, however.

\section{Late Systemic CS Treatment ( $>7$ Days of Life)}

The meta-analysis on late CS treatment ${ }^{7}$ included 21 RCTs enrolling a total of 1424 infants, almost invariably treated with dexamethasone. Overall, dexamethasone was associated with a lower mortality rate at 28 days (but not at 36 weeks PMA or later), and clear benefits in terms of respiratory health ( $\mathbf{- T a b l e ~} \mathbf{1}$ ). Adverse effects occurred mostly in the short term, including hyperglycemia, hypertension, and hypertrophic cardiomyopathy. There was also a higher rate of severe retinopathy of prematurity, but this was not associated with a higher rate of blindness (-Table $\mathbf{1}$ ). Dexamethasone was associated with more frequent abnormal neurological findings in four follow-up studies, but more robust data from 15 studies found no correlations between dexamethasone and either $\mathrm{CP}$ or major neurosensory disability (-Table 1). It is important to bear in mind, however, that several studies had relevant methodological limitations, and none of them was powered sufficiently to detect higher rates of adverse outcomes in the long term. The metaanalysis consequently concluded that late treatment with dexamethasone should be reserved for selected infants who cannot be weaned from mechanical ventilation, ${ }^{7}$ using the minimum necessary dose and the minimum duration of treatment, as already suggested in specific statements from the American Academy of Pediatrics and other scientific authorities. ${ }^{4}$

It is hard to say which infants might benefit from CS therapy, however. Data from a meta-regression analysis of 20 studies ${ }^{11}$ suggest that CS treatment may lower the risk of death/CP in infants at high risk ( $>50 \%$ ) of developing BPD, while the adverse effects outweigh the benefits in infants at lower risk of BPD. Unfortunately, estimating the risk of BPD in clinical practice is no easy matter either.

Given the concerns about neurotoxicity, dexamethasone must be used with caution. For this reason, there is a growing interest in the use of hydrocortisone, which might theoretically have a better safety profile due to its shorter half-life and specific action on CS receptors in the brain. ${ }^{4}$

Unfortunately, a recent, large RCT on late treatment with hydrocortisone for BPD (STOP-BPD study) yielded disappointing results. ${ }^{12}$ The trial enrolled 372 ventilator-dependent preterm infants ( $\mathrm{GA}<30$ weeks) given a 22-day course of hydrocortisone (cumulative dose: $72.5 \mathrm{mg} / \mathrm{kg}$ ) or placebo as of the second week of life. There was no difference in the combined primary outcome of death/BPD at 36 weeks PMA (relative risk [RR]: 0.87; 95\% confidence interval [CI]: 0.54$1.38 ; p=0.54$ ), despite earlier weaning from mechanical ventilation in the hydrocortisone group. Hyperglycemia requiring insulin therapy was the most important shortterm complication in the interventional arm of the study (18.2\% hydrocortisone-treated infants vs. $7.9 \%$ controls, $p=0.004)$. Data on any long-term adverse effects are not yet available.

Another important trial on late hydrocortisone treatment for BPD, sponsored by the NICHD (National Institute of Child Health and Human Development) Network, is still underway. ${ }^{13}$ This study plans to enroll 800 infants born $<30$ weeks GA, still ventilator-dependent beyond 14 days of life. Hydrocortisone will be administered over 10 days for a cumulative dose of $18 \mathrm{mg} / \mathrm{kg}$ (much smaller than in the STOP-BPD study). Primary outcome measures will be survival without moderate to severe BPD or moderate to severe neurodevelopmental impairment at 18 to 22 months corrected age. 
Table 1 Selected potential benefits and harms of systemic corticosteroids for BPD ${ }^{5-7}$

\begin{tabular}{|c|c|c|c|c|c|c|}
\hline \multicolumn{7}{|l|}{ Early treatment } \\
\hline & \multicolumn{3}{|l|}{ Dexamethasone } & \multicolumn{3}{|l|}{ Hydrocortisone } \\
\hline Benefits & Studies/Subjects & Effect size & & Studies/Subjects & Effect size & \\
\hline Mortality at 28 days & $16 / 2603$ & $1.06(0.90-1.24)$ & NS & $3 / 347$ & $0.78(0.50-1.23)$ & NS \\
\hline Mortality at 36 weeks & $14 / 2487$ & $1.01(0.89-1.14)$ & NS & $6 / 1246$ & $0.83(0.65-1.06)$ & NS \\
\hline BPD (28 days) & $16 / 2621$ & $0.85(0.79-0.92)$ & $p<0.001$ & $1 / 253$ & $1.00(0.85-1.18)$ & NS \\
\hline BPD (36 weeks) & $16 / 2584$ & $0.71(0.62-0.81)$ & $p<0.001$ & $8 / 1345$ & $0.91(0.80-1.05)$ & NS \\
\hline Death/BPD (28 days) & $14 / 2293$ & $0.91(0.86-0.96)$ & $p<0.001$ & $1 / 253$ & $1.00(0.90-1.12)$ & NS \\
\hline Death/BPD (36 weeks) & $16 / 2581$ & $0.87(0.80-0.94)$ & $p<0.001$ & 9/1379 & $0.90(0.82-0.99)$ & $p=0.05$ \\
\hline Severe ROP & $8 / 1507$ & $0.77(0.60-0.99)$ & $p=0.043$ & $6 / 1070$ & $0.87(0.63-1.21)$ & NS \\
\hline PDA & $17 / 2706$ & $0.76(0.69-0.84)$ & $p<0.001$ & $7 / 1307$ & $0.82(0.71-0.95)$ & $p<0.001$ \\
\hline \multicolumn{7}{|l|}{ Harms } \\
\hline Hyperglycemia & $12 / 2117$ & $1.35(1.21-1.49)$ & $p<0.001$ & $1 / 50$ & $0.92(0.50-1.67)$ & NS \\
\hline Hypertension & $11 / 1943$ & $1.84(1.53-2.21)$ & $p<0.001$ & $1 / 50$ & $3.0(0.33-26.92)$ & NS \\
\hline Gl bleeding & $10 / 1725$ & $1.87(1.35-2.58)$ & $p<0.001$ & $2 / 91$ & $1.53(0.27-8.74)$ & NS \\
\hline Gl perforation & 9/1936 & $1.73(1.20-2.51)$ & $p=0.004$ & $7 / 1104$ & $1.70(1.07-2.70)$ & $p=0.02$ \\
\hline $\mathrm{CP}$ & $7 / 921$ & $1.75(1.20-2.55)$ & $p=0.004$ & $6 / 1052$ & $1.05(0.66-1.66)$ & NS \\
\hline Death/CP & $7 / 921$ & $1.17(1.00-1.37)$ & $p=0.045$ & $6 / 1052$ & $0.86(0.71-1.05)$ & NS \\
\hline \multicolumn{7}{|l|}{ Late treatment } \\
\hline & \multicolumn{3}{|l|}{ Dexamethasone } & & & \\
\hline Benefits & Studies/Subjects & Effect size & & & & \\
\hline Mortality at 28 days & $8 / 656$ & $0.49(0.28-0.85)$ & $p=0.01$ & & & \\
\hline Mortality at 36 weeks & $7 / 360$ & $0.82(0.50-1.35)$ & NS & & & \\
\hline BPD (28 days) & $6 / 623$ & $0.87(0.81-0.94)$ & $p<0.001$ & & & \\
\hline BPD (36 weeks) & $11 / 580$ & $0.77(0.67-0.88)$ & $p<0.001$ & & & \\
\hline Death/BPD (28 days) & $5 / 563$ & $0.84(0.78-0.89)$ & $p<0.001$ & & & \\
\hline Death/BPD (36 weeks) & $11 / 580$ & $0.77(0.70-0.86)$ & $p<0.001$ & & & \\
\hline Extubation by day 7 & $15 / 761$ & $0.65(0.59-0.72)$ & $p<0.001$ & & & \\
\hline Discharge on $\mathrm{O}_{2}$ & $7 / 611$ & $0.71(0.54-0.94)$ & $p<0.001$ & & & \\
\hline \multicolumn{7}{|l|}{ Harms } \\
\hline Hyperglycemia & $17 / 1291$ & $1.51(1.26-1.81)$ & $p<0.001$ & & & \\
\hline Hypertension & $15 / 1235$ & $2.12(1.45-3.10)$ & $p<0.001$ & & & \\
\hline Gl bleeding & $7 / 992$ & $1.38(0.99-1.93)$ & NS & & & \\
\hline Gl perforation & $3 / 159$ & $1.60(0.28-9.31)$ & NS & & & \\
\hline Cardiomyopathy & $4 / 238$ & $2.76(1.33-5.74)$ & 0.006 & & & \\
\hline Severe ROP & $12 / 558$ & $1.38(1.07-1.79)$ & $p=0.01$ & & & \\
\hline Blindness & $13 / 784$ & $0.78(0.35-1.73)$ & NS & & & \\
\hline $\mathrm{CP}$ & $15 / 940$ & $1.10(0.79-1.54)$ & NS & & & \\
\hline Death/CP & $15 / 940$ & $0.93(0.77-1.12)$ & NS & & & \\
\hline
\end{tabular}

Abbreviations: BPD, bronchopulmonary dysplasia; CP, cerebral palsy; d, postnatal days; GI, gastrointestinal; NS, not specified; PDA, patent ductus arteriosus; ROP, retinopathy of prematurity; $w$, postmenstrual weeks.

In short, the routine early use of dexamethasone, though effective in preventing BPD, is not recommended due to its significant adverse effects, particularly the rate of CP. Cumulative data suggest that early hydrocortisone is not associated with significant neurotoxicity, but gastrointestinal perforation is a concern, and its efficacy in preventing
BPD is relatively limited. More data are needed before routine early hydrocortisone can be recommended. Late dexamethasone treatment confers a clear short-term respiratory benefit and is effective in preventing BPD; however, its safety at long term is not definitely established, so that its routine use remains contraindicated. Low total 
doses of dexamethasone may nonetheless be beneficial for a subpopulation of high-risk infants. The late use of hydrocortisone appears to be ineffectual, judging from currently available data. More information is awaited from studies currently underway.

\section{Inhaled Corticosteroids}

In the last two decades, concern about the risks of S-CS treatment for BPD has prompted interest in inhaled corticosteroids (I-CS), based on the assumption that topical treatments might theoretically lessen lung inflammation with fewer unwanted side effects.

Two recent meta-analyses reviewed the effects of ICS, again distinguishing between early ${ }^{14}$ and late treatment. ${ }^{15}$

\section{Early I-CS Treatment (Started at 0-14 Days of Life)}

The meta-analysis on early I-CS treatment ${ }^{14}$ included 10 studies and a total of 1,644 preterm infants. Although I-CS had no effect on BPD or mortality at 28 days or 36 postmenstrual weeks, the treatment did significantly reduce the rate of the combined outcome death/BPD (RR: $0.86,95 \% \mathrm{CI}$ : $0.75-0.99, p=0.04$ ), with no reported adverse effects. In addition, fewer of the surviving infants in the intervention arm had BPD at 36 weeks PMA. The authors warned, however, that this positive result should be interpreted with caution because the upper limit of the $\mathrm{CI}$ for the outcome death/BPD is infinity, which makes its clinical relevance questionable.

The results of the meta-analysis are clearly determined by the data from the largest RCT on I-CS, the "NEUROSIS" trial, ${ }^{16}$ in which early inhaled budesonide was tested on 863 newborns at high risk of BPD (23-27 weeks GA, requiring any level of positive pressure respiratory support). Compared with placebo budesonide significantly reduced BPD at 36 postmenstrual weeks (RR: 0.74; 95\%CI: 0.60-0.91; $p=0.004)$. This benefit was offset, however, by a worrying (though statistically not significant) increase in mortality ( 16.9 vs. $13.6 \%$; RR: 1.24 ; 95\% CI: $0.91-1.69 ; p=0.17$ ). At 18 to 22 months corrected age, this higher mortality in budesonide-treated participants was confirmed, this time achieving statistical significance (19.9 vs. $14.5 \%$; RR: $1.37,95 \% \mathrm{CI}$ : $1.01-1.86, p=0.04) .{ }^{17}$ The rates of long-term neurodevelopmental impairment were similar between budesonide and placebo groups.

Overall, the safety and effectiveness of early I-CS for the prevention of BPD remain uncertain, and further studies are warranted before they can be adopted in routine clinical practice.

\section{Late I-CS Treatment (Started at $\geq 7$ Days of Life)}

The meta-analysis on late I-CS treatment include eight trials involving 232 preterm infants receiving supplemental oxygen or on mechanical ventilation beyond the first week of life. ${ }^{15}$ I-CS yielded no benefits in terms of mortality, BPD, or the combined outcome death/BPD. Although a lower rate of extubation failure at 7 days emerged in the I-CS group, the duration of mechanical ventilation and oxygen dependence was unaffected. The limited number of participants and the considerable heterogeneity of the trials analyzed made it impossible to draw any firm conclusions regarding the safety and efficacy of late I-CS, so their clinical use is not currently recommended.

In short, direct nebulization of CS in the lung might potentially improve pulmonary inflammation while avoiding any systemic adverse effects. The early administration of inhaled budesonide seems to be effective in preventing BPD, but the serious issue regarding any increased mortality in treated infants needs to be clarified. No significant benefits have emerged to date from the sparse data currently available on treatment with I-CS starting beyond the first week of life.

\section{Intratracheal Administration of Corticosteroids}

Administering CS intratracheally, using surfactant as a vehicle, seems to be an attractive approach, theoretically ensuring a better delivery and distribution of CS inside the lungs. Yeh et $\mathrm{al}^{18}$ recently reported on an RCT in which 265 very low birth-weight infants with severe respiratory distress were randomized to receive either intratracheal budesonide combined with surfactant or surfactant alone within 4 hours of birth. The intervention group had a significantly lower incidence of the combined outcome death/BPD (42.0 vs. 66\%; RR: 0.58; 95\% CI: 0.44-0.77; $p<0.001$; NNT: $4.1 ; 95 \%$ CI: 2.8-7.8), with no short-term complications. The treatment was unassociated with any effects on neuromotor and cognitive function at a mean age of 30 months. A larger multicenter RCT designed by the same authors is currently underway. $^{19}$

In short, the intratracheal instillation of budesonide and surfactant is a promising option for preventing BPD, suitable for treating sick, intubated infants early in life. Currently available data point to a remarkable benefit, which-if replicated-would be clinically relevant.

\section{Conclusion}

The postnatal administration of CS to prevent or treat BPD is a powerful therapeutic option, but it is also associated with several side effects that may have a detrimental effect on the infant's health for life. The routine administration of S-CS is consequently not recommended at the present time. A renewed acceptance of a cautious, tailored use of S-CS is growing, while research efforts to identify less toxic preparations and therapeutic schedules and better methods for targeting candidates for treatment might improve the riskbenefit balance of CS.

Alternative methods of CS administration are becoming increasingly attractive too. Nebulized and intratracheally instilled CS seems promising, but more data are needed on their safety and efficacy.

Conflict of Interest

None declared. 


\section{References}

1 Stoll BJ, Hansen NI, Bell EF, et al; Eunice Kennedy Shriver National Institute of Child Health and Human Development Neonatal Research Network. Trends in care practices, morbidity, and mortality of extremely preterm neonates, 1993-2012. JAMA 2015; 314(10):1039-1051

2 Bonadies L, Zaramella P, Porzionato A, Muraca M, Baraldi E. Bronchopulmonary dysplasia: what's new on the horizon? Lancet Child Adolesc Health 2018;2(08):549-551

3 Baraldi E, Filippone M. Chronic lung disease after premature birth. N Engl J Med 2007;357(19):1946-1955

4 Watterberg KL; American Academy of Pediatrics. Committee on Fetus and Newborn. Policy statement-postnatal corticosteroids to prevent or treat bronchopulmonary dysplasia. Pediatrics 2010; 126(04):800-808

5 Doyle LW, Cheong JLY. Postnatal corticosteroids to prevent or treat bronchopulmonary dysplasia - who might benefit? Semin Fetal Neonatal Med 2017;22(05):290-295

6 Doyle LW, Cheong JL, Ehrenkranz RA, Halliday HL. Early ( $<8$ days) systemic postnatal corticosteroids for prevention of bronchopulmonary dysplasia in preterm infants. Cochrane Database Syst Rev 2017;10:CD001146

7 Doyle LW, Cheong JL, Ehrenkranz RA, Halliday HL. Late ( $>7$ days) systemic postnatal corticosteroids for prevention of bronchopulmonary dysplasia in preterm infants. Cochrane Database Syst Rev 2017;10:CD001145

8 Baud O, Maury L, Lebail F, et al; PREMILOC trial study group. Effect of early low-dose hydrocortisone on survival without bronchopulmonary dysplasia in extremely preterm infants (PREMILOC): a double-blind, placebo-controlled, multicentre, randomised trial. Lancet 2016;387(10030):1827-1836

9 Baud O, Trousson C, Biran V, Leroy E, Mohamed D, Alberti C; PREMILOC Trial Group. Association between early low-dose hydrocortisone therapy in extremely preterm neonates and neurodevelopmental outcomes at 2 years of age. JAMA 2017;317(13):1329-1337

10 Baud O, Trousson C, Biran V, Leroy E, Mohamed D, Alberti C; PREMILOC Trial group. Two-year neurodevelopmental outcomes of extremely preterm infants treated with early hydrocortisone: treatment effect according to gestational age at birth. Arch Dis Child Fetal Neonatal Ed 2019;104(01):F30-F35

11 Doyle LW, Halliday HL, Ehrenkranz RA, Davis PG, Sinclair JC. An update on the impact of postnatal systemic corticosteroids on mortality and cerebral palsy in preterm infants: effect modification by risk of bronchopulmonary dysplasia. J Pediatr 2014;165 (06):1258-1260

12 Onland W, Cools F, Kroon A, et al; STOP-BPD Study Group. Effect of hydrocortisone therapy initiated 7 to 14 days after birth on mortality or bronchopulmonary dysplasia among very preterm infants receiving mechanical ventilation: a randomized clinical trial. JAMA 2019;321(04):354-363

13 ClinicalTrialsgov. A randomized controlled trial of the effect of hydrocortisone on survival without bronchopulmonary dysplasia and on neurodevelopmental outcomes at 22-26 months of age in intubated infants $<30$ weeks gestation age. 2011 . Available at: https://clinicaltrials.gov/ct2/show/NCT01353313? term $=01353313$ \&rank=1. Accessed May 22, 2019

14 Shah VS, Ohlsson A, Halliday HL, Dunn M. Early administration of inhaled corticosteroids for preventing chronic lung disease in very low birth weight preterm neonates. Cochrane Database Syst Rev 2017;1:CD001969

15 Onland W, Offringa M, van Kaam A. Late ( $\geq 7$ days) inhalation corticosteroids to reduce bronchopulmonary dysplasia in preterm infants. Cochrane Database Syst Rev 2017;8:CD002311

16 Bassler D, Plavka R, Shinwell ES, et al; NEUROSIS Trial Group. Early inhaled budesonide for the prevention of bronchopulmonary dysplasia. N Engl J Med 2015;373(16):1497-1506

17 Bassler D, Shinwell ES, Hallman M, et al; Neonatal European Study of Inhaled Steroids Trial Group. Long-term effects of inhaled budesonide for bronchopulmonary dysplasia. N Engl J Med 2018;378(02):148-157

18 Yeh TF, Chen CM, Wu SY, et al. Intratracheal administration of budesonide/surfactant to prevent bronchopulmonary dysplasia. Am J Respir Crit Care Med 2016;193(01):86-95

19 ClinicalTrialsgov. Intratracheal budesonide/surfactant prevents BPD. Available at: https://clinicaltrials.gov/ct2/show/NCT03275415? term $=$ NCT03275415\&rank=1. Accessed May 22, 2019 